\title{
PREVALENCIA DE LA SINTOMATOLOGÍA EMOCIONAL Y COMPORTAMENTAL EN ADOLESCENTES ESPAÑOLES A TRAVÉS DEL STRENGTHS AND DIFFICULTIES QUESTIONNAIRE (SDQ)
}

\author{
Eduardo Fonseca-Pedrero, Mercedes Paino, Serafín Lemos-GirÁldeZ y José MuÑIZ \\ Departamento de Psicología, Universidad de Oviedo, Oviedo, Asturias \\ Centro de Investigación Biomédica en Red de Salud Mental, CIBERSAM, España
}

\begin{abstract}
Resumen: El objetivo del presente estudio fue examinar la prevalencia de los síntomas emocionales y comportamentales, a través del Strengths and Difficulties Questionnaire (SDQ), en una muestra representativa de adolescentes españoles. La muestra final la formaron un total de 1.319 estudiantes, 631 varones $(47,8 \%)$ y $688(52,2 \%)$ mujeres, pertenecientes a 28 centros escolares. La edad media fue de 15,70 años $(D T=1,02)$. Un porcentaje elevado de los participantes informó de algún problema emocional y/o comportamental. Asimismo, se encontraron diferencias estadísticamente significativas en función del sexo y la edad. Los resultados señalan que la adolescencia es una época del desarrollo donde la sintomatología afectiva y comportamental es bastante frecuente. Estos resultados tienen implicaciones en la comprensión de la psicopatología en la infancia y la adolescencia, así como de cara al establecimiento de centros asistenciales específicos y programas de prevención y detección temprana específicamente diseñados para este sector de la población.
\end{abstract}

Palabras clave: Prevalencia; adolescencia; SDQ; psicopatología; emocional, comportamental.

\section{Prevalence of emotional and behavioral symptoms in Spanish adolescents using the Strengths and Difficulties Questionnaire (SDQ)}

\begin{abstract}
The objective of the present study was to examine the prevalence of emotional and behavioral symptoms, using the Strengths and Difficulties Questionnaire (SDQ), in a representative sample of adolescents from the general Spanish population. The final sample was composed of a total of 1,319 students, 631 male (47.8\%) and $688(52.2 \%)$ female, attending 28 schools. The mean age was 15.70 years $(D T=1.02)$. A high percentage of participants reported some emotional and/ or behavioral problem. Statistically significant differences were found as a function of sex and age. Adolescence is a developmental stage where affective and behavioral symptomatology is fairly common. These results have implications for the comprehension of child and adolescent psychopathology as well as for the establishment of specific centers for assistance and prevention, and for early detection programs aimed at this specific sector of the population.
\end{abstract}

Keywords: Prevalence; adolescents; SDQ; psychopathology; behavioural; emotional.

La Encuesta Nacional de Salud España 2006, realizada a través del Strengths and Diffi-

Recibido: 7 julio 2010; aceptado 20 agosto 2010

Correspondencia: Eduardo Fonseca-Pedrero, Universidad de Oviedo, Facultad de Psicología, Plaza Feijoo s/n, 33003 Oviedo. Correo-e: efonseca@cop.es

Agradecimientos: Esta investigación ha sido financiada por el Ministerio de Ciencia e Innovación de España (MICINN), por el Instituto Carlos III, Centro de Investigación Biomédica en Red de Salud Mental (CIBERSAM). Referencias de los proyectos: BES-2006-12797, SEJ 200803934, PSI 2008-06220 y PSI 2008-03934. culties Questionnaire (SDQ) (Goodman, 1997) [Cuestionario de Capacidades y Dificultades], indicó que entre un 10 y un $20 \%$ de los niños y adolescentes españoles presentaba un trastorno mental, siendo éste además grave en el 4-6\% de los casos. Más aún, el 22,1\% de los niños y adolescentes de entre 4 y 15 años presentó riesgo de sufrir una alteración mental o del comportamiento (p. ej., problemas de conducta) (Ministerio de Sanidad y Política Social, 2006). Estudios epidemiológicos infanto-juveniles rea- 
lizados también en España arrojan tasas de prevalencia similares para diferentes problemas emocionales y comportamentales (FonsecaPedrero et al., 2010; Goméz-Beneyto, Bonet, Catalá, Puche y Vila, 1994; Polaino y Doménech, 1993; Sandoval, Lemos y Vallejo, 2006) siendo similares a las encontradas en población general (Bones et al., 2010; Haro et al., 2006). Estos resultados son convergentes con los encontrados en encuestas llevadas a cabo en otros países. Las tasas de prevalencia internacionales para los trastornos mentales en niños y adolescentes, clasificados según los criterios diagnósticos (DSM-IV y CIE-10), se sitúan entre el 5 y el 20\% de la población (Blanchard, Gurka y Blackman, 2006; Costello, Mustillo, Erkanli, Keeler y Angold, 2003; Ford, Goodman y Meltzer, 2003; Ravens-Sieberer et al., 2008; Simpson, Bloom, Cohen, Blumberg y Bourdon, 2005), siendo la tasa media del orden del 15\% (Roberts, Attkisson y Rosenblatt, 1998).

Como se puede observar un porcentaje elevado de niños y adolescentes presenta o presentará a lo largo de su vida un trastorno mental, ocasionando un claro impacto, no sólo en las esferas personal, académica, familiar y social, sino también a nivel económico y sanitario (Blanchard et al., 2006; Domino et al., 2009; Simpson et al., 2005). Además, muchos de los trastornos mentales parecen tener su comienzo en la niñez y/o adolescencia -aproximadamente un 50\% comienzan antes de los 15 años- y, en muchos casos, dicha sintomatología se mantiene estable hasta la edad adulta (Cohen, 2008; Costello et al., 2003; Kim-Cohen et al., 2003; Widiger, De Clercq y De Fruyt, 2009). Más aún, se debe tener en cuenta que la presencia de sintomatología afectiva y comportamental a nivel subclínico incrementa el riesgo posterior de desarrollar un trastorno mental de tipo severo (Cohen, Crawford, Johnson y Kasen, 2005; Klein, Shankman, Lewinsohn y Seeley, 2009; Najman et al., 2008; Welham et al., 2009).

En literatura previa se han utilizado diferentes entrevistas estructuradas y/o autoinformes para la valoración de la tasas de prevalencia de los problemas emocionales y comportamentales en población infanto-juvenil. Como es sabido, las entrevistas tienen algunas ventajas en comparación con los autoinformes como, por ejemplo, la realización de un diagnóstico formal, si bien entre sus principales desventajas se encuentra la necesidad de un profesional experto para su aplicación, y que su utilización suele ser lenta y costosa. En cambio, los autoinformes son fáciles y rápidos de administrar, sin embargo, no permiten realizar diagnósticos formales. En este sentido resulta de interés la utilización de instrumentos de medida como el SDQ (Goodman, 1997) con fines epidemiológicos, dada su brevedad (25 ítems), su fácil administración, la incorporación de facetas que miden aspectos positivos (p. ej., conducta prosocial), la valoración de síntomas frecuentes en adolescentes como son los problemas de conducta o de hiperactividad, y su utilidad como medida en contextos clínicos como, por ejemplo, para la valoración de resultados de tratamientos aplicados (Bourdon, Goodman, Rae, Simpson y Koretz, 2005; Goodman, 1999, 2001; Ruchkin, Jones, Vermeiren y SchwabStone, 2008; Vostanis, 2006).

La sintomatología emocional y comportamental evaluada mediante el SDQ parece variar en función del sexo y la edad de los adolescentes. En relación con el sexo, las mujeres obtienen mayores puntuaciones medias que los varones en síntomas emocionales y conducta prosocial; en cambio, los varones obtienen mayores puntuaciones medias que las mujeres en problemas de conducta, hiperactividad o problemas de relación (Giannakopoulos et al., 2009; Koskelainen, Sourander y Vauras, 2001; Muris, Meesters y van den Berg, 2003; Rønning, Handegaard, Sourander y Mørch, 2004; Svedin y Priebe, 2008; Van Roy, Grøholt, Heyerdahl y Clench-Aas, 2006; van Widenfelt, Goedhart, Treffers y Goodman, 2003; Yao et al., 2009). Con respecto a la edad, los resultados aún no son concluyentes. Algunos estudios encuentran una mayor sintomatología a medida que aumenta la edad (Giannakopoulos et al., 2009; Koskelainen et al., 2001; Rønning et al., 2004; Yao et al., 2009), mientras que otros encuentran una tendencia inversa (Muris et al., 2003; Van Roy et al., 2006) o no hallan ningún tipo de asociación (Prior, Virasinghe y Smart, 2005).

Hasta el momento, en España, se han llevado a cabo pocos estudios empíricos que traten de analizar y comprender la expresión de los problemas emocionales y comportamentales en 
población general adolescente no clínica. Dentro de este contexto epidemiológico de investigación, el principal objetivo del presente trabajo fue examinar las tasas de prevalencia de sintomatología emocional y comportamental en una muestra representativa de adolescentes de la población general española a través del Strengths and Difficulties Questionnaire (SDQ) (Goodman, 1997). Asimismo, se examinó el papel que desempeñan el sexo y la edad en la expresión de la sintomatología dentro de este sector de la población. El estudio de las tasas de prevalencia de síntomas emocional-comportamentales nos permite una mejor comprensión de la psicopatología infanto-juvenil y posibilita la mejora de los sistemas de salud pública a nivel de detección e intervención precoz, así como de tratamiento y gestión de recursos. De acuerdo con investigaciones previas, nos guía la hipótesis de que el porcentaje de adolescentes que informará de síntomas emocionales y comportamentales a nivel subclínico será elevado. Asimismo, se hipotetiza que la sintomatología comportamental y afectiva variará en función del sexo y la edad de los participantes.

\section{MÉTODO}

\section{Participantes}

La selección de los participantes se llevó a cabo mediante un muestreo aleatorio estratificado, por conglomerados, a nivel de aula, en una población aproximada de treinta y seis mil estudiantes pertenecientes a la comunidad autónoma del Principado de Asturias (región situada al norte de España). Los estratos se crearon en función de la zona geográfica (Oriente, Occidente, Central y Cuenca minera) y la etapa escolar (obligatoria y post-obligatoria). La probabilidad de elección de cada centro era directamente proporcional al número de alumnos correspondientes al mismo. Los alumnos pertenecían a diferentes centros escolares, públicos, concertados y privados, de Educación Secundaria Obligatoria y Ciclos de Formación Profesional. La muestra inicial fue de 1628 alumnos, si bien se eliminaron aquellos participantes que presentaban: (a) una puntuación elevada en la Escala Oviedo de Infrecuencia de Respuesta (INF-OV) (más de dos puntos) ( $n=$ 64); (b) dificultades de aprendizaje $(n=6)$; (c) una edad mayor de 17 años $(n=171)$; d) omisión de datos demográficos o un porcentaje elevado de ítems sin responder $(n=32)$; y e) puntuaciones outliers $(n=36)$. De este modo, la muestra final la formaron un total de 1319 estudiantes, 631 varones $(47,8 \%)$ y $688(52,2 \%)$ mujeres, pertenecientes a 28 centros escolares y 90 aulas. La edad media fue de 15,70 años $(D T=1,02)$, oscilando el rango de edad entre los 14 y los 17 años. La distribución en función de la edad para la muestra fue la siguiente: 14 años $(n=194 ; 14,7 \%), 15$ años $(n=357$; $27,1 \%), 16(n=411 ; 31,2 \%)$ años y 17 años $(n$ $=357 ; 27,1 \%)$.

\section{Instrumentos de evaluación}

Strengths and Difficulties Questionnaire (SDQ) (Goodman, 1997). Medida de autoinforme ampliamente utilizada para la valoración de diferentes problemas emocionales y comportamentales relacionados con la salud mental en niños y adolescentes, tomando como criterio los últimos 6 meses. El SDQ se compone de un total de 25 afirmaciones, que se distribuyen a lo largo de 5 subescalas (con 5 ítems cada una): Síntomas emocionales, Problemas de conducta, Hiperactividad, Problemas de relación y Conducta prosocial. Las primeras cuatro subescalas forman una puntuación total de Dificultades. En este estudio se utilizó un formato de respuesta Likert de 5 opciones de respuesta (1 "Completamente en desacuerdo"; 5 "Completamente de acuerdo"), por lo que la puntuación de cada subescala oscila entre 5 y 25 puntos, y la puntuación total entre 20 y 100 . Es digno de mención que en estudios previos se ha utilizado la versión Likert de 3 puntos del SDQ, no obstante la literatura psicométrica previa indica que un formato de respuesta Likert de 5 opciones mejora la fiabilidad de las puntuaciones, la obtención de evidencias de validez y la satisfacción de los usuarios (Lozano, García-Cueto y Muñiz, 2008; Muñiz, Fidalgo, García-Cueto, Martínez y Moreno, 2005; Muñiz, García-Cueto y Lozano, 2005). En todas las subescalas, a excepción 
de Conducta prosocial, una mayor puntuación es indicativa de mayor sintomatología afectiva y/o comportamental. Las propiedades psicométricas del SDQ en su versión autoinformada se encuentran ampliamente analizadas, relativas tanto a la fiabilidad, como a diferentes evidencias de validez de estructura interna, convergente-discriminante y predicitiva (Bourdon et al., 2005; Goodman, 1999, 2001; Ruchkin et al., 2008; Vostanis, 2006). En estudio se ha utilizado la versión adaptada y traducida al español disponible en internet (http://www.sdqinfo.com) (García et al., 2000). En esta investigación los niveles de consistencia interna para las subescalas del SDQ oscilaron entre 0,58 (Problemas de conducta) y 0,71 (Síntomas emocionales).

Escala Oviedo de Infrecuencia de Respuesta (INF-OV) (Fonseca-Pedrero, Lemos-Giráldez, Paino, Villazón-García y Muñiz, 2009). Es un instrumento de autoinforme compuesto por 12 ítems en formato tipo Likert de 5 categorías (1 "Completamente en desacuerdo"; 5 "Completamente de acuerdo") que se ha desarrollado siguiendo las directrices para la construcción de tests (Schmeiser y Welch, 2006) y de ítems de elección múltiple (Moreno, Martínez y Muñiz, 2006). Su objetivo es detectar a aquellos participantes que responden de forma azarosa, pseudoazarosa o deshonesta en autoinformes (incluye para ello ítems de respuesta verdadera única, p. ej., "La distancia entre Madrid y Barcelona es mayor que entr e Madrid y Nue va York'). Los alumnos que puntúan más de tres ítems de forma incorrecta se eliminan de la participación en el estudio.

\section{Procedimiento}

La administración del cuestionario se llevó a cabo de forma colectiva, en grupos de 10 a 35 estudiantes, durante el horario escolar y en aulas acondicionadas para este fin. El estudio fue presentado a los participantes como una investigación sobre las diversas características de la personalidad, asegurándoles la confidencialidad de sus respuestas, así como del carácter voluntario de su participación. La administración del cuestionario se realizó en todo momento bajo la supervisión de un investigador. En los casos necesarios se recogió el consentimiento paterno. Este estudio se encuadra dentro de una investigación más amplia sobre detección e intervención temprana en los trastornos psicológicos en la adolescencia.

\section{RESULTADOS}

\section{Estadísticos descriptivos}

En la Tabla 1 se presentan las puntuaciones medias y desviaciones típicas para los ítems del SDQ, tanto para la muestra total, como en función del sexo y dos grupos de edad (grupo de 14-15 años y grupo de 16-17 años). La media de la puntuación total de Dificultades del SDQ para la muestra total fue de $46,05(D T=9,03)$. El rango de puntuaciones osciló entre 22 y 86 puntos. En el caso de los varones, la puntuación total de Dificultades osciló también entre 22 y 86 , y en las mujeres entre 23 y 75 . En función de la edad, la puntuación del grupo de 14-15 años osciló entre 22 y 73 , y en el grupo de 16-17 años, entre 24 y 86.

\section{Prevalencia de los síntomas emocionales y comportamentales en los adolescentes}

En la Tabla 2 se recogen número y porcentaje de participantes para la muestra total, para hombres y mujeres separadamente, y en función de los dos grupos de edad, que puntuaron 4 ó 5 ("De acuerdo" o "Totalmente de acuer$d o ")$ en las opciones de respuesta de los ítems que componen el SDQ. Como se puede observar, un porcentaje considerable de los participantes informó de síntomas afectivos y comportamentales. Sin ir más lejos, el 34,6\% de la muestra refirió estar preocupado a menudo (ítem 8), y el 25,7\% alegó perder el control cuando se enfada (ítem 7). Del mismo modo, el $15,7 \%$ de la muestra puntuó 4 ó 5 en el ítem 13 (Me siento a menudo triste, desanimado o con ganas de llorar), y el 47,1\% en el ítem 15 (Me distraigo con facilidad, me cuesta concentrarme). Los ítems que obtuvieron mayores tasas de respuestas afirmativas en la muestra total fueron el ítem 15 (Me distraigo con faci- 
Tabla 1. Estadísticos descriptivos (medias y desviaciones típicas) para los ítems del Strengths and Difficulties Questionnaire (SDQ)

\begin{tabular}{|c|c|c|c|c|c|c|c|c|c|c|}
\hline \multirow[b]{2}{*}{ Ítems $S D Q$} & \multicolumn{2}{|c|}{$\begin{array}{l}\text { Total } \\
(N=1.319)\end{array}$} & \multicolumn{2}{|c|}{$\begin{array}{l}\text { Hombre } \\
(n=631)\end{array}$} & \multicolumn{2}{|c|}{$\begin{array}{c}\text { Mujer } \\
(n=688)\end{array}$} & \multicolumn{2}{|c|}{$\begin{array}{c}14-15 \text { años } \\
(n=551)\end{array}$} & \multicolumn{2}{|c|}{$\begin{array}{c}16-17 \text { años } \\
(n=768)\end{array}$} \\
\hline & $M$ & $D T$ & $M$ & $D T$ & $M$ & $D T$ & $M$ & $D T$ & $M$ & $D T$ \\
\hline $\begin{array}{l}\text { 3. Suelo tener muchos dolores de cabeza, estómago o } \\
\text { náuseas }\end{array}$ & 2,01 & 1,12 & 1,73 & 0,91 & 2,27 & 1,23 & 1,96 & 1,08 & 2,06 & 1,15 \\
\hline 8. A menudo estoy preocupado & 2,99 & 1,15 & 2,93 & 1,13 & 3,05 & 1,16 & 2,89 & 1,18 & 3,07 & 1,12 \\
\hline $\begin{array}{l}\text { 13. Me siento a menudo triste, desanimado o con ganas } \\
\text { de llorar }\end{array}$ & 2,15 & 1,18 & 1,89 & 1,09 & 2,39 & 1,22 & 2,19 & 1,16 & 2,13 & 1,19 \\
\hline $\begin{array}{l}\text { 16. Me pongo nervioso con las situaciones nuevas, } \\
\text { fácilmente pierdo la confianza en mí mismo }\end{array}$ & 2,90 & 1,27 & 2,68 & 1,21 & 3,10 & 1,30 & 2,83 & 1,32 & 2,95 & 1,24 \\
\hline 24. Tengo muchos miedos, me asusto fácilmente & 2,06 & 1,10 & 1,77 & 0,98 & 2,32 & 1,13 & 2,02 & 1,07 & 2,08 & 1,11 \\
\hline $\begin{array}{l}\text { 5. Cuando me enfado, me enfado mucho y pierdo el } \\
\text { control }\end{array}$ & 2,74 & 1,21 & 2,84 & 1,19 & 2,64 & 1,21 & 2,64 & 1,22 & 2,80 & 1,19 \\
\hline 7. Por lo general soy obediente $*$ & 2,42 & 0,95 & 2,46 & 0,96 & 2,37 & 0,93 & 2,45 & 1,01 & 2,39 & 0,91 \\
\hline $\begin{array}{l}\text { 12. Peleo con frecuencia con otros, manipulo a los } \\
\text { demás }\end{array}$ & 1,62 & 0,92 & 1,78 & 1,02 & 1,48 & 0,79 & 1,56 & 0,88 & 1,67 & 0,94 \\
\hline 18. A menudo me acusan de mentir o de hacer trampas & 1,93 & 1,05 & 2,18 & 1,13 & 1,70 & 0,91 & 1,97 & 1,10 & 1,90 & 1,02 \\
\hline $\begin{array}{l}\text { 22. Cojo cosas que no son mías de casa, la escuela o } \\
\text { de otros sitios }\end{array}$ & 1,66 & 0,95 & 1,82 & 1,02 & 1,51 & 0,85 & 1,65 & 0,93 & 1,66 & 0,96 \\
\hline jien solitario y tiendo a jugar solo & 1,68 & 1,06 & 1,76 & 1,09 & 1,60 & 1,01 & 1,61 & 1,04 & 1,72 & 1,06 \\
\hline 11. Tengo un buen amig & 1,28 & 0,69 & 1,32 & 0,76 & 1,24 & 0,61 & 1,30 & 0,74 & 1,26 & 0,65 \\
\hline 14. Por lo gene & 2,05 & 0,79 & 1,99 & 0,80 & 2,11 & 0,78 & 2,05 & 0,81 & 2,05 & 0,79 \\
\hline $\begin{array}{l}\text { 19. Otra gente de mi edad se mete conmigo o se burla } \\
\text { de mí }\end{array}$ & 1,78 & 1,01 & 1,84 & 1,05 & 1,72 & 0,98 & 1,90 & 1,08 & 1,70 & 0,96 \\
\hline 23. Me llevo mejor con adultos que con otros de mi edad & 2,45 & 1,15 & 2,40 & 1,12 & 2,50 & 1,18 & 2,23 & 1,11 & 2,61 & 1,16 \\
\hline $\begin{array}{l}\text { 2. Soy inquieto, hiperactivo, no puedo permanecer } \\
\text { quieto por mucho tiempo }\end{array}$ & 2,83 & 1,22 & 2,88 & 1,27 & 2,78 & 1,17 & 2,70 & 1,21 & 2,92 & 1,22 \\
\hline 10. Estoy todo el tiempo moviéndome, me muevo de- & 2,83 & 1,17 & 2,86 & 1,22 & 2,79 & 1,13 & 2,71 & 1,18 & 2,90 & 1,16 \\
\hline distraioo con facilidad me cuesta concentrarme & 3,31 & 1,23 & 3,40 & 1,23 & 3,21 & 1,22 & 3,25 & 1,26 & 3,35 & 1,20 \\
\hline 21. Pienso las cosas antes de hacerlas* & 2,50 & 1,07 & 2,49 & 1,05 & 2,51 & 1,10 & 2,52 & 1,09 & 2,49 & 1,06 \\
\hline 25. Termino lo que empiezo, tengo buena concentración* & 2,87 & 1,07 & 2,89 & 1,09 & 2,85 & 1,06 & 2,83 & 1,10 & 2,90 & 1,05 \\
\hline $\begin{array}{l}\text { 1. Procuro ser agradable con los demás. Tengo en cuen- } \\
\text { ta los sentimientos de las otras personas }\end{array}$ & 4,30 & 0,73 & 4,19 & 0,76 & 4,39 & 0,69 & 4,33 & 0,70 & 4,27 & 0,75 \\
\hline $\begin{array}{l}\text { 4. Normalmente comparto con otros mis juguetes, lá- } \\
\text { pices, etc. }\end{array}$ & 4,14 & 0,83 & 3,97 & 0,85 & 4,28 & 0,78 & 4,12 & 0,85 & 4,15 & 0,81 \\
\hline 9. Ayudo si alguien está enfermo, disgustado o herido & 4,15 & 0,80 & 3,98 & 0,84 & 4,31 & 0,73 & 4,11 & 0,85 & 4,19 & 0,77 \\
\hline 17. Trato bien a los niños más pequeños & 4,36 & 0,87 & 4,26 & 0,89 & 4,46 & 0,85 & 4,40 & 0,87 & 4,34 & 0,88 \\
\hline 20. A menudo me ofrezco para ayudar (a padres, maes- & 3,36 & 0,97 & 3,25 & 0,97 & 3,45 & 0,96 & 3,36 & 0,98 & 3,36 & 0,96 \\
\hline
\end{tabular}

Nota. ${ }^{*}$ Los ítems 7, 11, 14, 21 y 25 han sido recodificados de acuerdo con el formato de corrección.

Síntomas emocionales: ítems 3, 8, 13, 16 y 24; Problemas de conducta: ítems 5, 7, 12, 18 y 22; Problemas de relación: ítems 6, 11 , 14, 19 y 23; Hiperactividad ítems: 2, 10 15, 21 y 25; (mayores puntuaciones indican mayor gravedad); Prosocial ítems 1, 4, 9, 17 y 20 (a mayor puntuación mejor conducta prosocial).

lidad, me cuesta concentrarme) y el ítem 8 ( $A$ menudo estoy preocupado). Los ítems que obtuvieron una menor tasa de respuestas afirmativas fueron el ítem 11 (Tengo un buen amigo por lo menos) y el ítem 14 (Por lo general cai- go bien a la otr a gente de mi edad) - estos ítems se deben interpretar en el sentido contrario, ya que en función de los criterios de corrección del SDQ han sido recodificados-; por ejemplo, el ítem 7 "Por lo general soy obedien- 
te" indica, una vez recodificado, que el 11,8\% de la muestra total, por lo general, no es obediente. En relación con la subescala Conducta prosocial del SDQ, una mayor puntuación es indicativa de una mejor conducta prosocial, por lo que los cinco últimos ítems de la Tabla 2 se deben interpretar en este sentido. El porcentaje de respuestas afirmativas para los ítems que conforman esta subescala oscilaron en la muestra total entre el $44,6 \%$ y $89,6 \%$.

Tabla 2. Número y porcentaje de participantes de la muestra total, de hombres y mujeres separadamente, y por grupos de edad, que puntuaron 4 ó 5 en los ítems del Strengths and Difficulties Questionnaire (SDQ)

\begin{tabular}{|c|c|c|c|c|c|c|c|c|c|c|}
\hline \multirow[b]{2}{*}{ Ítems $S D Q$} & \multicolumn{2}{|c|}{$\begin{array}{c}\text { Total } \\
(n=1.319)\end{array}$} & \multicolumn{2}{|c|}{$\begin{array}{l}\text { Hombre } \\
(n=631)\end{array}$} & \multicolumn{2}{|c|}{$\begin{array}{c}\text { Mujer } \\
(n=688)\end{array}$} & \multicolumn{2}{|c|}{$\begin{array}{c}14-15 \text { años } \\
(n=551)\end{array}$} & \multicolumn{2}{|c|}{$\begin{array}{l}16-17 \text { años } \\
(n=768)\end{array}$} \\
\hline & $n$ & $\%$ & $n$ & $\%$ & $n$ & $\%$ & $n$ & $\%$ & $n$ & $\%$ \\
\hline $\begin{array}{l}\text { 3. Suelo tener muchos dolores de cabeza, estómago o } \\
\text { náuseas }\end{array}$ & 154 & 11,7 & 36 & 5,7 & 118 & 17,2 & 56 & 10,2 & 98 & 12,8 \\
\hline 8. A menudo estoy preocupado & 456 & 34,6 & 204 & 32,3 & 252 & 36,6 & 171 & 31,0 & 285 & 37,1 \\
\hline $\begin{array}{l}\text { 13. Me siento a menudo triste, desanimado o con ganas } \\
\text { de llorar }\end{array}$ & 207 & 15,7 & 66 & 10,5 & 141 & 20,5 & 83 & 15,1 & 124 & 16,1 \\
\hline $\begin{array}{l}\text { 16. Me pongo nervioso con las situaciones nuevas, } \\
\text { fácilmente pierdo la confianza en mí mismo }\end{array}$ & 452 & 34,3 & 163 & 25,8 & 289 & 42,0 & 175 & 31,8 & 277 & 36,1 \\
\hline 24. Tengo muchos miedos, me asusto fácilmente & 153 & 11,6 & 46 & 7,3 & 107 & 15,6 & 54 & 9,8 & 99 & 12,9 \\
\hline $\begin{array}{l}\text { 5. Cuando me enfado, me enfado mucho y pierdo el } \\
\text { control }\end{array}$ & 339 & 25,7 & 179 & 28,4 & 160 & 23,3 & 127 & 23,0 & 212 & 27,6 \\
\hline 7. Por lo general soy obediente* & 156 & 11,8 & 84 & 13,3 & 72 & 10,5 & 72 & 13,1 & 84 & 10,9 \\
\hline 12. Peleo con frecuencia con otros, manipulo a los demás & 64 & 4,9 & 46 & 7,3 & 18 & 2,6 & 22 & 4,0 & 42 & 5,5 \\
\hline 18. A menudo me acusan de mentir o de hacer trampas & 128 & 9,7 & 88 & 13,9 & 40 & 5,8 & 61 & 11,1 & 67 & 8,7 \\
\hline $\begin{array}{l}\text { 22. Cojo cosas que no son mías de casa, la escuela o } \\
\text { de otros sitios }\end{array}$ & 75 & 5,7 & 47 & 7,4 & 28 & 4,1 & 29 & 5,3 & 46 & 6,0 \\
\hline 6. Soy más bien solitario y tiendo a jugar solo & 117 & 8,9 & 63 & 10,0 & 54 & 4,8 & 48 & 8,7 & 69 & 9,0 \\
\hline 11. Tengo un buen amigo por lo $\mathrm{m}$ & 30 & 2,3 & 20 & 3,2 & 10 & 1,5 & 13 & 2,4 & 17 & 2,2 \\
\hline 14. Por lo general caigo bien a la otra gente de mi edad* & 50 & 3,8 & 27 & 4,3 & 23 & 3,3 & 23 & 4,2 & 27 & 3,5 \\
\hline $\begin{array}{l}\text { 19. Otra gente de mi edad se mete conmigo o se burla } \\
\text { de mí }\end{array}$ & 97 & 7,4 & 54 & 8,6 & 43 & 6,3 & 54 & 9,8 & 43 & 5,6 \\
\hline 23. Me llevo mejor con adultos que con otros de mi edad & 218 & 16,5 & 90 & 14,3 & 128 & 18,6 & 64 & 11,6 & 154 & 20,1 \\
\hline $\begin{array}{l}\text { 2. Soy inquieto, hiperactivo, no puedo permanecer } \\
\text { quieto por mucho tiempo }\end{array}$ & 389 & 29,5 & 207 & 32,8 & 182 & 26,5 & 142 & 25,8 & 247 & 32,2 \\
\hline $\begin{array}{l}\text { 10. Estoy todo el tiempo moviéndome, me muevo } \\
\text { demasiado }\end{array}$ & 364 & 27,6 & 194 & 30,7 & 170 & 24,7 & 137 & 24,9 & 227 & 29,6 \\
\hline 15. Me distraigo con facilidad, me cuesta concentrarme & 621 & 47,1 & 317 & 50,2 & 304 & 44,2 & 248 & 45,0 & 373 & 48,6 \\
\hline 21. Pienso las cosas antes de hacerlas* & 212 & 16,1 & 98 & 15,5 & 114 & 16,6 & 89 & 16,2 & 123 & 16,0 \\
\hline 25. Termino lo que empiezo, tengo buena concentración* & 357 & 27,1 & 175 & 27,7 & 182 & 26,5 & 148 & 26,9 & 209 & 27,2 \\
\hline $\begin{array}{l}\text { 1. Procuro ser agradable con los demás. Tengo en } \\
\text { cuenta los sentimientos de las otras personas }\end{array}$ & 1.182 & 89,6 & 552 & 87,5 & 630 & 91,6 & 506 & 91,8 & 676 & 88,0 \\
\hline $\begin{array}{l}\text { 4. Normalmente comparto con otros mis juguetes, } \\
\text { lápices, etc. }\end{array}$ & 1072 & 81,3 & 474 & 75,1 & 598 & 86,9 & 434 & 78,8 & 638 & 83,1 \\
\hline 9. Ayudo si alguien está enfermo, disgustado o herido & 1096 & 83,1 & 480 & 76,1 & 616 & 89,5 & 437 & 79,3 & 659 & 85,8 \\
\hline 17. Trato bien a los niños más pequeños & 1156 & 87,6 & 545 & 86,4 & 611 & 88,8 & 482 & 87,5 & 674 & 87,8 \\
\hline $\begin{array}{l}\text { 20. A menudo me ofrezco para ayudar (a padres, } \\
\text { maestros, niños) }\end{array}$ & 588 & 44,6 & 256 & 40,6 & 332 & 48,3 & 242 & 43,9 & 346 & 45,1 \\
\hline
\end{tabular}

*Los ítems 7, 11, 14, 21 y 25 han sido recodificados de acuerdo con el formato de corrección.

**Síntomas emocionales: ítems 3, 8, 13, 16 y 24; Problemas de conducta: ítems 5, 7, 12, 18 y 22; Problemas de relación: ítems 6, 11, 14, 19 y 23; Hiperactividad ítems: 2, 10 15, 21 y 25; (mayores puntuaciones indican mayor gravedad); Prosocial ítems 1, 4, 9, 17 y 20 (a mayor puntuación mejor conducta prosocial). 
Expresión de los síntomas emocionales y comportamentales en función del sexo y la edad

A continuación, se examinó la posible influencia del sexo y la edad en la expresión de la sintomatología emocional y comportamental de los adolescentes evaluados. Para ello se llevó a cabo un análisis multivariado de la varianza (MANOVA), tomando con factores fijos el sexo y la edad (recodificada en dos grupos en función de su nivel de escolarización: 14-15 años y 1617 años), y como variables dependientes las subescalas y la puntuación total de Dificultades del SDQ. Se utilizó el valor Lambda de Wilks para observar si existían diferencias significativas en todas las variables dependientes tomadas en conjunto. Como índice de tamaño del efecto se empleó el estadístico eta cuadrado parcial ( $\eta^{2}$ parcial). En la Tabla 3 se presentan las medias y las desviaciones típicas en las subescalas y la puntuación total del SDQ para los 4 grupos. El valor $\lambda$ de Wilks reveló la presencia de diferencias estadísticamente significativas en función del sexo (Wilks $\lambda=0,830, p<0,001$ ), no así en función de la edad (Wilks $\lambda=0,993, p=$ 0,085). En relación con el sexo, se encontraron diferencias estadísticamente significativas en las subescalas Síntomas emocionales, Problemas de conducta, Hiperactividad y Conducta prosocial. Las mujeres obtuvieron mayores puntuaciones que los varones en la subescalas Síntomas emocionales $\left(F=94,68, p<0,001, \eta^{2}\right.$ parcial $=0,067)$ y Conducta prosocial $(F=$ $66,81, p<0,001, \eta^{2}$ parcial $\left.=0,048\right)$, en cambio los varones obtuvieron una mayor puntuación media en las subescalas Problemas de conducta $\left(F=4,29, p=0,039, \eta^{2}\right.$ parcial $\left.=0,003\right) \mathrm{e}$ Hiperactividad $\left(F=79,01, p<0,001, \eta^{2}\right.$ parcial $=0,057)$ en comparación con las mujeres. En función de la edad, y aunque el valor Lambda de Wilks no resultó ser estadísticamente significativo, sí se encontraron diferencias estadísticamente significativas en la subescala Hiperactividad $\left(F=6,08, p=0,014, \eta^{2}\right.$ parcial $\left.=0,005\right)$ y en la puntuación total de Dificultades del SDQ $\left(F=6,78, p=0,009, \eta^{2}\right.$ parcial $\left.=0,005\right) . \mathrm{El}$ grupo de 14-15 años puntúo más bajo que el grupo de 15-16 años. Asimismo, en la subescala de Conducta prosocial se encontró una interacción estadísticamente significativa entre el sexo y la edad $\left(F=4,66, p=0,031, \eta^{2}\right.$ parcial $=0,004)$.

\section{DISCUSIÓN Y CONCLUSIONES}

El principal objetivo del presente trabajo fue examinar las tasas de prevalencia de la sintomatología emocional y comportamental autoinformada en una muestra representativa de adolescentes españoles a través del Strengths and Difficulties Questionnaire (SDQ) (Goodman, 1997). Asimismo, se examinó en el papel que desempeñan el sexo y la edad en la expresión fenotípica de este tipo de sintomatología. Los resultados indicaron que un elevado porcentaje de los adolescentes evaluados informó de síntomas emocionales y comportamentales a nivel subclínico, y que la expresión de dicha

Tabla 3. Comparaciones entre las puntuaciones medias en las subescalas y puntuación total del Strengths and Difficulties Questionnaire (SDQ) en función del sexo y la edad

\begin{tabular}{|c|c|c|c|c|}
\hline$S D Q$ & $\begin{array}{c}\text { Varones } \\
(n=631) \\
\text { Media }(D T)\end{array}$ & $\begin{array}{c}\text { Mujeres } \\
(n=688) \\
\text { Media }(D T)\end{array}$ & $\begin{array}{c}14-15 \text { años } \\
(n=551) \\
\text { Media }(D T)\end{array}$ & $\begin{array}{c}16-17 \text { años } \\
(n=768) \\
\text { Media }(D T)\end{array}$ \\
\hline Síntomas emocionales & $11,01(3,52)$ & $13,14(4,11)$ & $11,88(4,00)$ & $12,30(3,96)$ \\
\hline Problemas de conducta & $11,09(3,27)$ & $9,70(2,83)$ & $10,27(3,11)$ & $10,43(3,14)$ \\
\hline Hiperactividad & $14,53(3,92)$ & $14,15(3,66)$ & $14,01(3,73)$ & $14,55(3,82)$ \\
\hline Problemas de relación & $9,31(2,99)$ & $9,17(2,76)$ & $9,10(2,80)$ & $9,34(2,91)$ \\
\hline Conducta prosocial & $19,66(2,72)$ & $20,91(2,51)$ & $20,32(2,77)$ & $20,30(2,63)$ \\
\hline Puntuación total de Dificultades* & $45,94(9,16)$ & $46,16(8,93)$ & $45,27(9,01)$ & $46,62(9,02)$ \\
\hline
\end{tabular}

* La puntuación total de Dificultades es la suma de todas las subescalas del SDQ a excepción de la subescala Conducta prosocial 
sintomatología varió en función del sexo y la edad de los participantes.

Considerando la muestra total de participantes, los resultados mostraron que entre el 11,6 y el 34,6\% de los adolescentes presentó algún síntoma de tipo emocional y que entre el 16,1 y el $47,1 \%$ informó de un comportamiento hiperactivo. Del mismo modo, entre el 2,3\% y el $16,5 \%$ de los participantes presentó algún problema de relación y entre el 4,9 y el $25,7 \%$ de los adolescentes presentó algún problema del comportamiento. Las tasas de sintomatología afectivo-comportamental encontradas en este estudio son bastante similares a las recogidas en estudios nacionales e internacionales tanto en población infanto-juvenil como en población adulta. Sin ir más lejos, en la Encuesta Nacional de Salud España 2006, se encontró que entre un 10 y un $20 \%$ de los niños y adolescentes españoles presentaba un trastorno mental. La tasa media de prevalencia para los trastornos mentales clasificados según los criterios diagnósticos (DSM-IV y CIE-10) en niños y adolescentes en estudios internacionales se sitúan en el 15\% (Roberts et al., 1998), oscilando según los estudios entre el 5 y el $20 \%$ de la población (Blanchard et al., 2006; Costello et al., 2003; Ford et al., 2003; Ravens-Sieberer et al., 2008; Simpson et al., 2005). Estos resultados señalan que los problemas emocionales y comportamentales son un fenómeno psicológico bastante común en población infanto-juvenil, y que a pesar de la diversidad de estudios, muestras e instrumentos de medida utilizados, los resultados son bastante convergentes.

En consonancia con la literatura previa, se encontró que la expresión de la sintomatología emocional y comportamental, evaluada mediante el SDQ, variaba en función del sexo y la edad de los adolescentes (Giannakopoulos et al., 2009; Koskelainen et al., 2001; Muris et al., 2003; Rønning et al., 2004; Svedin y Priebe, 2008; Van Roy et al., 2006; van Widenfelt et al., 2003; Yao et al., 2009). En este estudio los resultados indicaron que las mujeres obtuvieron mayores puntuaciones medias que los varones en la subescalas Síntomas emocionales y Conducta prosocial del SDQ; en cambio, los varones obtuvieron mayores puntuaciones en las subescalas Problemas de conducta e Hiperactividad. Por ejemplo,
Rønning et al. (2004), utilizando una muestra de 4167 adolescentes noruegos, encontraron resultados totalmente convergentes con los hallados en este trabajo. Asimismo, datos similares se hallan cuando se examina el papel del sexo utilizando la versión del SDQ para padres y/o profesores (Capron, Théorond y Duyme, 2007; Muris et al., 2003). Los datos encontrados en el presente estudio en relación con la edad indican que el grupo de 15-16 años presentó una puntuación media mayor que el grupo de 13-14 años en la subescala Hiperactividad y en la puntuación total de Dificultades del SDQ. Resultados similares se han encontrado en investigaciones previas (Giannakopoulos et al., 2009; Koskelainen et al., 2001; Rønning et al., 2004; Yao et al., 2009) Por ejemplo, Giannakopoulos et al. (2009), utilizando una muestra de 1194 adolescentes griegos, encontraron que el grupo de 15-17 años presentaba una mayor puntuación en la subescalas Hiperactividad y Problemas de conducta del SDQ, en comparación con el grupo de 11-14 años; no obstante, otros trabajos han encontrado que los más jóvenes obtienen una puntuación mayor cuando se les compara con los de mayor edad (Muris et al., 2003; Van Roy et al., 2006) $\mathrm{o}$ incluso no encuentran ningún tipo de asociación (Prior et al., 2005). Los datos parecen aún no ser totalmente concluyentes por lo que se debe seguir profundizando en el papel que desempeña la edad en la expresión fenotípica de este tipo de sintomatología afectiva y comportamental.

Una considerable información científica está basada en el estudio epidemiológico de la psicopatología infantil y adolescente en los últimos veinte años. La evidencia de que la salud mental de niños y adolescentes es un área que merece que continúe siendo objeto de atención científica y de Salud Pública está bien documentada por organizaciones internacionales (National Institute of Mental Health, 2001; U.S. Department of Health and Human Services, 2001), y los costes económicos asociados con la psicopatología infantil y adolescente subrayan la necesidad de datos y métodos epidemiológicos útiles en este sector de edad (Buka, Monuteaux y Earls, 2002). Las investigaciones epidemiológicas en psicopatología infantil tienen 3 objeti- 
vos fundamentales (Kellam y Ensminger, 1980): a) para los propósitos de Salud Pública, la epidemiología proporciona información importante relativa a la prevalencia de los trastornos, utilización de servicios, resultados de tratamientos y costes; b) dado que la epidemiología estudia la distribución, determinantes y causas de los trastornos, las investigaciones epidemiológicas tienen como segundo objetivo avanzar en el entendimiento del origen y curso de la psicopatología a edades tempranas, lo que incluye entender el significado de las circunstancias tempranas, tanto biológicas como sociales, que contribuyen al origen y progresión de los trastornos mentales; c) estos datos idealmente llevan también a la tercera gran aplicación de la epidemiología, el diseño y evaluación de estrategias preventivas y de tratamiento.

Los resultados encontrados en el presente estudio deberían interpretarse a la luz de las siguientes limitaciones. En primer lugar, la edad es un factor relevante a tener en cuenta en la expresión fenomenológica de estos síntomas. La adolescencia es un periodo madurativo del desarrollo donde se dan una serie de cambios a diferentes niveles (biológico, afectivo, cognitivo y social) que podrían estar jugando un rol importante en los resultados del estudio. En segundo lugar, existe la problemática inherente a la aplicación de cualquier tipo de autoinforme, como son las posibles dificultades en la interpretación y comprensión de ítems por parte de algunos participantes, así como por las tasas de falsos positivos, por lo que hubiese sido interesante la utilización de informantes externos, como padres o profesores, vía autoinforme hetero-aplicado. En tercer lugar, no hay que perder de vista la naturaleza transversal de esta investigación, que imposibilita el establecimiento de inferencias causa-efecto. En cuarto lugar, es cabe mencionar que en estudios previos donde se utiliza el SDQ se ha utilizado la versión Likert de 3 puntos, mientras que en esta investigación se ha utilizado una modificación de la versión original, por lo que se debe ser cauteloso en cuanto a la comparación entre estudios.

Futuros estudios deberían examinar la relación de los problemas afectivos y comporta- mentales con otros autoinformes (Fonseca-Pedrero, Paino et al., 2009; Sandín, Valiente y Chorot, 2009) y/o variables clínicas (Frías, Carrasco, Fernández, García y García, 2009; Iglesias y Romero, 2009) que puedan ayudar en la prevención y detección precoz. Del mismo modo, resulta de gran interés llevar a cabo estudios longitudinales en muestras representativas de adolescentes españoles, de cara a determinar el papel predictor de los problemas emocionales y comportamentales en el desarro1lo hacia un trastorno psicológico en la etapa adulta, favoreciendo de este modo el establecimiento de marcadores de riesgo o factores protectores, así como la implementación de programas de prevención y detección temprana.

\section{REFERENCIAS}

Blanchard, L. T., Gurka, M. J., y Blackman, J. A. (2006). Emotional, developmental, and behavioral health of American children and their families: a report from the 2003 National Survey of Children's Health. Pediatrics, 117, e1202-1212.

Bones Rocha, K., Pérez, K., Rodríguez-Sanz, M., Borrell, C. y Obiols, J. E. (2010). Prevalencia de problemas de salud mental y su asociación con variables socioeconómicas, de trabajo y salud: resultados de la Encuesta Nacional de Salud de España. Psicothema, 22, 389395.

Bourdon, K. H., Goodman, R., Rae, D. S., Simpson, G. y Koretz, D. S. (2005). The Strengths and Difficulties Questionnaire: U.S. normative data and psychometric properties. Journal of American Academy of Child and Adolescent Psychiatry, 44, 557-564.

Buka, S. L., Monuteaux, M. y Earls, F. (2002). The Epidemiology of Child and Adolescent Mental Disorders. En M. T. Tsuang y M. Tohen (Eds.), Textbook in psychiatry epidemiology (pp. 629-656). New York: Willey-Liss.

Capron, C., Théorond, C. y Duyme, M. (2007). Psychometric properties of the French version of the self-report and teacher Strengths and Difficulties Questionnaire (SDQ) European Journal of Psyc hological Assessment, 23, 79-88.

Cohen, P. (2008). Child development and personality disorder. Psychiatric Clinics of North America 31, 477-493.

Cohen, P., Crawford, T. N., Johnson, J. G. y Kasen, S. (2005). The children in the community study of developmental course of personality disorder. Journal of Personality Disorders, 19, 466-486.

Costello, E. J., Mustillo, S., Erkanli, A., Keeler, G. y Angold, A. (2003). Prevalence and development of psy- 
chiatric disorders in childhood and adolescence. Archives of General Psychiatry, 60, 837-844.

Domino, M. E., Burns, B. J., Mario, J., Reinecke, M. A., Vitiello, B., Weller, E. B., Kratochvil, C. J., May, D. E., Feeny, N. C., Robins, M., Hallin, M. J., Silva, S. G. y March, J. S. (2009). Service use and costs of care for depressed adolescents: who uses and who pays? Journal of Clinical Child \& Adolescent Psychology, 38, 826-836.

Fonseca-Pedrero, E., Lemos-Giráldez, S., Paino, M., Villazón-García, U. y Muñiz, J. (2009). Validation of the Schizotypal Personality Questionnaire Brief form in adolescents. Schizophrenia Research, 111, 53-60.

Fonseca-Pedrero, E., Paino, M., Lemos-Giráldez, S., Villazón-García, U., García-Cueto, E., Bobes, J. y Muñiz, J. (2009). Versión reducida del Cuestionario TPSQ de Estilos Perceptuales y de Pensamiento. Psicothema, 21, 499-505.

Fonseca-Pedrero, E., Wells, C., Paino, M., Lemos-Giráldez, S., Villazón-García, U., Sierra, S., García-Portilla González, M. P., Bobes, J. y Muñiz, J. (2010). Measurement invariance of the Reynolds Depression Adolescent Scale across gender and age. International Journal of Testing, 10, 133-148.

Ford, T., Goodman, R. y Meltzer, H. (2003). The British Child and Adolescent Mental Health Survey 1999: the prevalence of DSM-IV disorders. Journal of the American Academy of Child andAdolescent Psychiatry 42, 1203-1211.

Frías, A., Carrasco, A., Fernández, M. J., García, A. y García, B. (2009). Comorbilidad entre trastornos de conducta y depresión mayor en la adolescencia: prevalencia, teorías explicativas y estatus nosológico Revista de Psicopatología y Psicología Clínica, 14, 1-16.

García, P., Goodman, R., Mazaira, J., Torres, A., Rodríguez-Sacristán, J., Hervas, A. y Fuentes, J. (2000). El Cuestionario de Capacidades y Dificultades. Revista de Psiquiatría Infanto-Juvenil, 1, 12-17.

Giannakopoulos, G., Tzavara, C., Dimitrakaki, C., Kolaitis, G., Rotsika, V. y Tountas, Y. (2009). The factor structure of the Strengths and Difficulties Questionnaire (SDQ) in Greek adolescents. Annals of General Psychiatry, 8, 20.

Goméz-Beneyto, M., Bonet, A., Catalá, M. A., Puche, E. y Vila, V. (1994). The prevalence of child psychiatric disorders in the city of Valencia. Acta Psychiatrica Scandinavica, 89, 352-357.

Goodman, R. (1997). The strengths and difficulties questionnaire: a research note. Journal of Child Psychology and Psychiatry, 38, 581-586.

Goodman, R. (1999). The extended version of the Strengths and Difficulties Questionnaire as a guide to child psychiatric caseness and consequent burden. Journal of Child Psychology and Psychiatry, 40, 791-801.
Goodman, R. (2001). Psychometric properties of the strengths and difficulties questionnaire. Journal of American Academy of Child andAdolescent Psychiatry, 40, 1337-1345

Haro, J. M., Palacín, C., Vilagut, G., Martínez, M., Luque, I., Codony, M., Dolz, M. y Alonso, J. (2006). Prevalencia de los trastornos mentales y factores asociados: resultados del estudio ESEMeD-España. Medicina clínica, 126, 445-451.

Iglesias, B. y Romero, E. (2009). Estilos parentales percibidos, psicopatología y personalidad en la adolescencia. Revista de Psicopatología y Psicología Clínica, 14, 63-77.

Kellam, S. G. y Ensminger, M. E. (1980). Theory and method in child psychiatric epidemiology. En F. Earls (Ed.), Studies of Children (pp. 145-180). New York: Prodist.

Kim-Cohen, J., Caspi, A., Moffitt, T. E., Harrington, H., Milne, B. J. y Poulton, R. (2003). Prior juvenile diagnoses in adults with mental disorder: developmental follow-back of a prospective-longitudinal cohort. Archives of General Psychiatry, 60, 709-717.

Klein, D. N., Shankman, S. A., Lewinsohn, P. M. y Seeley, J. R. (2009). Subthreshold depressive disorder in adolescents: predictors of escalation to full-syndrome depressive disorders. Journal of American Academy of Child and Adolescent Psychiatry, 48, 703-710.

Koskelainen, M., Sourander, A. y Vauras, M. (2001). Selfreported strengths and difficulties in a community sample of Finnish adolescents. European Child \& Adolescent Psychiatry, 10, 180-185.

Lozano, L. M., García-Cueto, E. y Muñiz, J. (2008). Effect of the number of response categories on the reliability and validity of rating scales. Methodology, 4, 73-79.

Ministerio de Sanidad y Política Social. (2006). Encuesta Nacional de Salud de España 2006 . Descargado el 29/06/2010; http://www.msc.es/estadEstudios/estadisticas/encuestaNacional/encuesta2006.htm.

Moreno, R., Martínez, R. y Muñiz, J. (2006). New guidelines for developing multiple-choice items. Methodo$\log y, 2,65-72$.

Muñiz, J., Fidalgo, A. M., García-Cueto, E., Martínez, R. y Moreno, R. (2005). Análisis de los items. Madrid: La Muralla.

Muñiz, J., García-Cueto, E. y Lozano, L. M. (2005). Item format and the psychometric properties of the Eysenck Personality Questionnaire. Personality and Individual Differences, 38, 61-69.

Muris, P., Meesters, C. y van den Berg, F. (2003). The Strengths and Difficulties Questionnaire (SDQ)--further evidence for its reliability and validity in a community sample of Dutch children and adolescents. European Child \& Adolescent Psychiatry, 12, 1-8.

Najman, J. M., Heron, M. A., Hayatbakhsh, M. R., Dingle, K., Jamrozik, K., Bor, W., O'Callaghan, M. J. y 
Williams, G. M. (2008). Screening in early childhood for risk of later mental health problems: A longitudinal study. Journal of Psychiatric Research, 42, 694-700.

National Institute of Mental Health. (2001). Blueprint for Change: Research on Children and Adolescent Mental Health. Washington, DC: National Advisory Mental Health Council Workgroup on Child and Adolescent Mental Health Intervention Development and Deployment.

Polaino, A. y Doménech, E. (1993). Prevalence of childhood depression: results of the firts study in Spain. Journal of Child Psychology and Psychiatry, 34, 10071017.

Prior, M., Virasinghe, S. y Smart, D. (2005). Behavioural problems in Sri Lankan schoolchildren. Social Psychiatry and Psychiatric Epidemiology, 40, 654-662.

Ravens-Sieberer, U., Wille, N., Erhart, M., Bettge, S., Wittchen, H. U., Rothenberger, A., Herpertz-Dahlmann, B., Resch, F., Hölling, H., Bullinger, M., Barkmann, C., Schulte-Markwort, M. y Döpfner, M. (2008). Prevalence of mental health problems among children and adolescents in Germany: results of the BELLA study within the National Health Interview and Examination Survey. European Child \& Adolescent Psychiatry, 17, 22-33.

Roberts, R. E., Attkisson, C. C. y Rosenblatt, A. (1998). Prevalence of psychopathology among children and adolescents. American Journal of P sychiatry, 155, 715-725.

Rønning, J. A., Handegaard, B. H., Sourander, A. y Mørch, W. T. (2004). The Strengths and Difficulties Self-Report Questionnaire as a screening instrument in Norwegian community samples. European Child \& Adolescent Psychiatry, 13, 73-82.

Ruchkin, V., Jones, S., Vermeiren, R. y Schwab-Stone, M. (2008). The Strengths and Difficulties Questionnaire: The Self-Report Version in American Urban and Suburban Youth. Psychological Assessment, 20, 175-182.

Sandín, B., Valiente, R. M. y Chorot, P. (2009). RCADS: evaluación de los síntomas de ansiedad y depresión en niños y adolescentes Revista de Psicopatología y Psicología Clínica, 14, 193-206.

Sandoval, M., Lemos, S. y Vallejo, G. (2006). Self-reported competences and problems in Spanish adolescents: A normative study of the YSR. Psicothema, 18, 804809.

Schmeiser, C. B. y Welch, C. (2006). Test development. En R. L. Brennan (Ed.), Educational Measurement (4th ed.) (pp. 307-353). Westport, CT: American Council on Education/Praeger.

Simpson, G. A., Bloom, B., Cohen, R. A., Blumberg, S. y Bourdon, K. H. (2005). U. S. Children with Emotional and Behavioural Difficulties: Data from the 2001, 2002, and 2003 National Health Interview Surveys. Advance Data Form Vital and Health Statistics, $360,1-13$.

Svedin, C. G. y Priebe, G. (2008). The Strengths and Difficulties Questionnaire as a screening instrument in a community sample of high school seniors in Sweden. Nordic Journal of Psychiatry, 62, 225-232.

U.S. Department of Health and Human Services. (2001). Report of the Surgeon Generals Conference on Childrens Mental Health: A National Action Agenda. Rockville, MD: U.S. Department of Health and Human Services, Substance Abuse and Mental Health Services Administration, Center for Mental Health Services, National Institute of Health, National Institute of Mental Health.

Van Roy, B., Grøholt, B., Heyerdahl, S. y Clench-Aas, J. (2006). Self-reported strengths and difficulties in a large Norwegian population 10-19 years : age and gender specific results of the extended SDQ-questionnaire. European Child \& Adolescent Psychiatry, 15, 189-198.

van Widenfelt, B. M., Goedhart, A. W., Treffers, P. D. y Goodman, R. (2003). Dutch version of the Strengths and Difficulties Questionnaire (SDQ). European Child \& Adolescent Psychiatry, 12, 281-289.

Vostanis, P. (2006). Strengths and Difficulties Questionnaire: research and clinical applications. Current Opinion in Psychiatry, 19, 367-372.

Welham, J., Scott, J., Williams, G., Najman, J., Bor, W., O'Callaghan, M. y McGrath, J. (2009). Emotional and behavioural antecedents of young adults who screen positive for non-affective psychosis: a 21-year birth cohort study. Psychological Medicine, 39, 625-634.

Widiger, T. A., De Clercq, B. y De Fruyt, F. (2009). Childhood antecedents of personality disorder: An alternative perspective. Development and Psychopathology, 21, 771-791.

Yao, S., Zhang, C., Zhu, X., Jing, X., McWhinnie, C. M. y Abela, J. R. (2009). Measuring adolescent psychopathology: psychometric properties of the self-report strengths and difficulties questionnaire in a sample of Chinese adolescents. Journal of Adolescent Health, $45,55-62$. 